\title{
Efecto del etiquetado de semáforo en el contenido nutricional y el consumo de bebidas gaseosas en Ecuador
}

\author{
Victor Peñaherrera, ${ }^{1}$ Carlos Carpio, ${ }^{2}$ Luis Sandoval, ${ }^{1}$ Marcos Sánchez, ${ }^{3}$ Tania Cabrera, ${ }^{4}$ \\ Patricia Guerrero ${ }^{5}$ e Ivan Borja ${ }^{6}$
}

Forma de citar

Peñaherrera V, Carpio C, Sandoval L, Sánchez M, Cabrera T, Guerrero P, et al. Efecto del etiquetado semáforo en el contenido nutricional y el consumo de bebidas gaseosas en Ecuador. Rev Panam Salud Publica. 2018;42:e177. https://doi.org/10.26633/RPSP.2018.177

RESUMEN Objetivo. El objetivo de este estudio fue evaluar el efecto del etiquetado de semáforo nutricional en las compras de bebidas gaseosas y en su contenido nutricional en Ecuador.

Métodos. Se utilizaron dos bases de datos: la primera fue proporcionada por la Agencia Nacional de Regulación, Control y Vigilancia Sanitaria del Ecuador y la segunda se obtuvo de la empresa internacional de investigación de mercados Kantar World Panel. Se hizo un análisis antes-después de la introducción del etiquetado de semáforo con pruebas $t$ de Student para evaluar cambios en las medias del contenido nutricional y de las compras de bebidas gaseosas y se usaron métodos de regresión lineal múltiple para evaluar cambios en las compras debido a la introducción del etiquetado de semáforo.

Resultados. El consumo promedio per capita de bebidas gaseosas se redujo después del etiquetado en 0,003 L/mes, que es un valor de pequeña magnitud en relación con el consumo promedio per capita $(1,678 \mathrm{~L} / \mathrm{mes})$. Los resultados de las pruebas $t$ de Student indicaron que esta diferencia no fue estadísticamente diferente de cero. Además, con los análisis de regresión tampoco se encontraron pruebas empíricas de que el etiquetado de semáforo haya afectado el consumo de bebidas gaseosas. Con relación al contenido de azúcar, se observó una reducción promedio de $0,93 \mathrm{gr} / 100 \mathrm{ml}$ de bebida.

Conclusiones. No se encontraron pruebas empíricas de que la implementación del etiquetado de semáforo haya cambiado los hábitos de compra de bebidas gaseosas en Ecuador, pero sí de que disminuyó el contenido de azúcar de las bebidas gaseosas en el país.

Palabras clave Bebidas gaseosas; obesidad; política de salud; Ecuador.

Se estima que al menos 2,8 millones de personas en el mundo mueren cada año a

\footnotetext{
Departamento de Administración de Agronegocios, Universidad Zamorano, Tegucigalpa, Honduras. La correspondencia se debe dirigir a Luis Sandoval. Correo electrónico: lsandoval@zamorano.edu

2 Departamento de Economía Agrícola, Universidad de Texas Tech, Texas, Estados Unidos de América.
}

causa de enfermedades asociadas con las epidemias de obesidad y sobrepeso,

\footnotetext{
Centro Internacional para la Excelencia de la Industria Alimentaria (ICFIE), Universidad de Texas Tech, Texas, Estados Unidos de América.

4 Universidad Nacional de Loja, Loja, Ecuador.

5 Escuela de Medicina, Universidad Nacional de Loja, Loja, Ecuador.

Universidad San Francisco de Quito, Quito, Ecuador.
}

como las enfermedades cardiovasculares, la hipertensión, la diabetes y el cáncer (1, 2). Aunque inicialmente estas epidemias solo afectaban a países de altos ingresos, hoy en día en los países de medianos e incluso de bajos ingresos se padecen estas enfermedades (2). Por ejemplo, en Ecuador, considerado un país de medianosaltos ingresos (3), la prevalencia de

Este es un articulo de acceso abierto distribuido bajo los términos de la licencia Creative Commons Attribution-NonCommercial-NoDerivs 3.0 IGO, que permite su uso, distribución y reproducción en cualquier medio, siempre que el trabajo original se cite de la manera adecuada. No se permiten modificaciones a los artículos ni su uso comercial. Al reproducir un artículo no debe haber ningún indicio de que la OPS o el artículo avalan a una organización o un producto específico. El uso del logo de la OPS no está permitido. Esta leyenda debe conservarse, junto con la URL original del artículo. 
obesidad y sobrepeso es alta: en 2012, $29,9 \%$ de niños en edad escolar, 26,0\% de adolescentes y $62,8 \%$ de adultos en el país padecían obesidad o sobrepeso (4).

Varios países del mundo, incluidos Reino Unido, Australia, Holanda, Chile y Ecuador han tomado la iniciativa de combatir la tendencia creciente de la obesidad y el sobrepeso de su población mediante políticas públicas que buscan influir en sus hábitos de consumo. En el caso de Ecuador, el Gobierno aprobó el 29 de noviembre de 2013 una nueva Norma Sanitaria para el Etiquetado de Alimentos Procesados. Esta nueva norma tiene como propósito regular el etiquetado de los alimentos procesados para consumo humano distribuidos en el país con el objetivo de facilitar la interpretación del contenido nutricional de los alimentos a los consumidores (5).

La Norma Sanitaria para el Etiquetado de Alimentos Procesados, adoptada por el Ministerio de Salud Pública del Ecuador, incluye un sistema de etiquetado de semáforo para los productos procesados que complementa la Etiqueta de Información Nutricional (EIN) convencional. La EIN indica las cantidades de nutrientes en una porción del alimento y su contribución porcentual a una dieta estándar diaria de 2000 calorías (6). Por otro lado, el etiquetado de semáforo nutricional usa tres luces de semáforo para indicar si el contenido de grasa, azúcar y sal es alto (rojo), medio (amarillo) o bajo (verde) (5). La categorización de los productos se basa en rangos de contenidos nutricionales por peso o volumen especificados en los reglamentos técnicos de la regulación según se muestra en el cuadro 1 (5). Los productos con semáforos color verde se presentan como una opción más saludable en comparación con aquellos con semáforos amarillos y rojos, puesto que contienen niveles más bajos de grasa, azúcar y sal. De este modo, se busca disminuir el

CUADRO 1. Contenidos nutricionales por peso y volumen especificados en los reglamentos técnicos de la regulación

\begin{tabular}{llll}
\hline \multirow{2}{*}{ Componente } & \multicolumn{3}{c}{ Concentración } \\
\cline { 2 - 4 } & \multicolumn{1}{c}{ Baja } & \multicolumn{1}{c}{ Media } & \multicolumn{1}{c}{ Alta } \\
\hline Grasa total & $\leq 3 \mathrm{~g} / 100 \mathrm{~g}$ & entre 3 y $20 \mathrm{~g} / 100 \mathrm{~g}$ & $\geq 20 \mathrm{~g} / 100 \mathrm{~g}$ \\
& $\leq 1,5 \mathrm{~g} / 100 \mathrm{ml}$ & entre $1,5 \mathrm{y} 10 \mathrm{~g} / 100 \mathrm{ml}$ & $\geq 10 \mathrm{~g} / 100 \mathrm{ml}$ \\
Azúcar & $\leq 5 \mathrm{~g} / 100 \mathrm{~g}$ & entre 5 y $15 \mathrm{~g} / 100 \mathrm{~g}$ & $\geq 15 \mathrm{~g} / 100 \mathrm{ml}$ \\
& $\leq 2,5 \mathrm{~g} / 100 \mathrm{ml}$ & entre 2,5 y $7,5 \mathrm{gr} / 100 \mathrm{ml}$ & $\geq 7,5 \mathrm{~g} / 100 \mathrm{ml}$ \\
& $\leq 120 \mathrm{mg} / 100 \mathrm{~g}$ & entre 120 y $600 \mathrm{mg} / 100 \mathrm{~g}$ & $\geq 600 \mathrm{mg} / 100 \mathrm{~g}$ \\
Sal (Sodio) & $\leq 120 \mathrm{mg} / 100 \mathrm{ml}$ & entre 120 y $600 \mathrm{mg} / 100 \mathrm{ml}$ & $\geq 600 \mathrm{mg} / 100 \mathrm{ml}$ \\
\hline
\end{tabular}

Fuente: referencia 5. consumo de los alimentos altos en azúcar y grasa (causantes de la obesidad y el sobrepeso) y de alimentos altos en sodio (causantes de la hipertensión).

Aunque a escala internacional hay estudios que revelan que el etiquetado de semáforo facilita la comprensión de los consumidores de la información nutricional presentada en las EIN convencionales, hay muchas menos pruebas empíricas respecto al efecto del uso del semáforo en el cambio de los hábitos de consumo de la población. En el Reino Unido (7) y en Australia (8) se evaluaron las percepciones de los consumidores de varios modelos de etiquetado de semáforo frontal empleados para indicar los niveles de grasa, azúcar y sodio presentes en varios alimentos. En ambos estudios se observó que los consumidores entendieron correctamente los parámetros nutricionales del etiquetado de semáforo. El efecto de este etiquetado en las decisiones de compra de alimentos también se ha evaluado en el Reino Unido (9) y en Australia (10), donde se examinaron las diferencias en ventas de alimentos calificados como saludables y no saludables antes y después de la implementación del etiquetado de semáforo en varios supermercados. En ninguno de los estudios se encontraron diferencias entre las ventas de los alimentos antes y después de la implementación del etiquetado.

Con relación a estudios que evalúan el etiquetado de semáforo en Ecuador, la bibliografía es aún muy limitada. Cuatro estudios llaman la atención, los tres primeros por evaluar cualitativamente el efecto del semáforo, de los cuales dos son tesis de maestría $(11,12)$, y el cuarto por ser una revisión bibliográfica. De los estudios cualitativos, en el primero se evaluó la percepción de estudiantes universitarios de la ciudad de Quito con respecto al etiquetado de semáforo (11); en el segundo se evaluó la percepción y utilización del etiquetado de semáforo en consumidores de jugos y bebidas de frutas procesadas en varios supermercados en la ciudad de Quito (12), y en el tercero, la percepción de los consumidores de diferentes grupos de edad y lugar de residencia y de ambos sexos mediante la técnica de los grupos focales (13). En general, en los tres estudios se encontró que los consumidores reconocen la presencia del etiquetado de semáforo y saben utilizarlo, pero que esto no influye significativamente en su decisión de compra $(11,12)$ a menos que ya tengan estilos de vida saludables (13). Estos estudios son de naturaleza cualitativa y no evalúan el efecto del etiquetado utilizando datos de compras de alimentos. El cuarto estudio hace referencia a un análisis cualitativo de la variación del volumen de productos lácteos atribuyendo algunas de las variaciones al etiquetado de semáforo sin considerar ninguna otra variable que haya podido afectar el consumo (14). No se ha encontrado ningún estudio que evalué la respuesta de la industria al etiquetado de semáforo, específicamente en relación con cambios en la formulación y composición nutricional de los alimentos.

Debido a la ausencia de bibliografía en que se evalúe el efecto del etiquetado semáforo en los hábitos de consumo y la formulación de alimentos por parte de la industria, los objetivos de esta investigación fueron: 1) evaluar el efecto del etiquetado de semáforo en el comportamiento de compra de bebidas gaseosas de los consumidores ecuatorianos y 2) evaluar el efecto del etiquetado de semáforo en el contenido nutricional de las bebidas gaseosas.

El énfasis del presente estudio recae en las bebidas gaseosas por las siguientes razones. Primero, el consumo de bebidas gaseosas se considera alto en Ecuador (4). Segundo, las bebidas gaseosas están entre el grupo de productos que, según el criterio de los investigadores, son más criticados por su asociación con el sobrepeso. Por último, como el único nutriente que cambia es el azúcar, se simplifica la interpretación de los resultados.

\section{MATERIALES Y MÉTODOS}

En este estudio se utilizaron dos bases de datos. La primera fue adquirida de la empresa internacional de investigación de mercados Kantar World Panel, que contiene información de compra de alimentos y bebidas (volumen y precios) de un panel de 1646 hogares ecuatorianos 
representativos de la población tanto en su distribución geográfica como en su nivel socioeconómico. La información de compras se encuentra agregada mensualmente, de enero de 2013 a diciembre de 2015. Los datos están disponibles agregados mensualmente para todos los hogares y también por grupos socioeconómicos (alto, bajo y medio). La segunda base de datos se obtuvo de la Agencia Nacional de Regulación, Control y Vigilancia Sanitaria (ARCSA) y contiene información del contenido de azúcar de las bebidas gaseosas antes y después de la introducción del etiquetado de semáforo. De la base de datos de Kantar se utilizaron 22 bebidas gaseosas para evaluar el efecto del etiquetado de semáforo en los hábitos de consumo y de la base de datos de ARCSA, 14 para evaluar la respuesta de la industria respecto al contenido de azúcar de las bebidas gaseosas (no todas las bebidas de la base de datos Kantar estuvieron disponibles en la base de datos del ARCSA). El período de enero de 2013 a agosto de 2014 se consideró como el período antes de la introducción del etiquetado de semáforo y el período de septiembre 2014 a diciembre 2015, como el período después de la introducción del etiquetado de semáforo, ya que la fecha límite para la adopción del etiquetado de semáforo para compañías medianas y grandes (que producen todas las bebidas de mayor consumo en el país) fue el 29 de agosto del 2014. Es importante destacar que este estudio se limita a poco más de un año tras la implementación del etiquetado de semáforo para poder evaluar el efecto del etiquetado de semáforo por sí solo. No se utilizó un período más largo, ya que a partir de 2016 se aprobó el impuesto a las bebidas gaseosas y metodológicamente es imposible aislar completamente el efecto del etiquetado de semáforo del efecto del impuesto.

Para comparar medias, se utilizaron dos pruebas $\mathrm{t}$ de Student. La primera evalúa si hay diferencias en el consumo promedio de bebidas gaseosas antes y después de la introducción del etiquetado de semáforo. Para calcular las diferencias de los períodos antes y después, se usaron los valores de consumo per capita mensual de cada gaseosa en esos períodos de tiempo con la siguiente fórmula:

Cambio en consumo $=$ consumo después - consumo antes

Con la segunda prueba $t$, se evalúa si hay diferencias en el contenido de azúcar de las bebidas gaseosas ambas antes y después de la introducción del etiquetado de semáforo. El cambio en el contenido de azúcar para cada bebida gaseosa se calculó con la siguiente fórmula:

Cambio en el contenido de azúcar = contenido después - contenido antes

considerando que el efecto esperado es una reducción en el contenido de azúcar de las bebidas ante la posibilidad de una reducción en la demanda debido a la presencia de etiquetas rojas de contenido de azúcar.

Además de las pruebas t, se construyeron tres modelos de regresión lineal múltiple para evaluar el efecto dela introducción del etiquetado de semáforo en el consumo de tres grupos de gaseosas: 1) todas las bebidas gaseosas, 2) bebidas gaseosas altas en azúcar y 3) bebidas gaseosas bajas en azúcar. Los modelos de regresión permiten controlar los resultados por otros factores que pudiesen haber afectado también el consumo de bebidas gaseosas además del etiquetado de semáforo. La especificación de estos modelos se basó en la teoría económica de la demanda (15). Por lo tanto, además de una variable dicotómica que indica la introducción del semáforo, los modelos de regresión incluyen como variables explicativas precios, variables dicotómicas de estacionalidad, variables que indican el nivel socioeconómico de los grupos de consumidores y una variable de tiempo (mes). Los modelos se pueden expresar así:

Todas la bebidas gaseosas:

$\log ($ QtotalG $)=\beta_{0}+\beta_{1} \log (\mathrm{IPFG})+\beta_{2}$ $\mathrm{TL}+\beta_{3} \mathrm{~T}+\beta_{4}$ Alto $+\beta_{5}$ Medio $+\beta_{6} \mathrm{~T} 1+\beta_{7}$ $\mathrm{T} 2+\beta_{8} \mathrm{~T} 3$

\section{Altas en azúcar:}

$\log ($ QtotalAA $)=\beta_{0}+\beta_{1} \log ($ IPFBA $)+$ $\beta_{2} \log ($ IPFAA $)+\beta_{3} \mathrm{TL}+\beta_{4} \mathrm{~T}+\beta_{5}$ Alto $+\beta_{6}$ Medio $+\beta_{7} \mathrm{~T} 1+\beta_{8} \mathrm{~T} 2+\beta_{9} \mathrm{~T} 3$

Bajas en azúcar:

$\log ($ QtotalBA $)=\beta_{0}+\beta_{1} \log ($ IPFBA $)+\beta_{2}$ $\log ($ IPFAA $)+\beta_{3} \mathrm{TL}+\beta_{4} \mathrm{~T}+\beta_{5}$ Alto $+\beta_{6}$ Medio $+\beta_{7} \mathrm{~T} 1+\beta_{8} \mathrm{~T} 2+\beta_{9} \mathrm{~T} 3$

donde:

$\log ($.$) representa el logaritmo natural$ de una variable.

QtotalG es el consumo per capita en litros de bebidas gaseosas.

QtotalAA es el consumo per capita en litros de bebidas gaseosas altas en azúcar.
QtotalBA es el consumo per capita en litros de bebidas gaseosas bajas en azúcar.

IPFG es el índice de precio Fisher (IPF) de todas las bebidas gaseosas.

IPFAA es el IPF de las bebidas gaseosas altas en azúcar.

IPFBA es el IPF de las bebidas gaseosas bajas en azúcar.

TL es una variable dicotómica que indica la presencia del etiquetado de semáforo.

T es una variable de tiempo (mes).

Alto y Medio son variables dicotómicas que expresan los niveles socioeconómicos alto y medio, respectivamente.

T1, T2 y T3 son variables dicotómicas que indican los tres primeros trimestres del año (estacionalidad).

\section{Cálculo del índice de precio Fisher}

El índice de precio ideal Fisher (IPF) es la media geométrica de los índices Laspeyres (L) y Paasche (P). Habitualmente, este índice de precio se utiliza para calcular una medida estandarizada de los precios de un grupo de productos (16). En este caso se utilizó para calcular el precio de grupos de todas las bebidas gaseosas, de las altas en azúcar y de las bajas en azúcar. La fórmula para calcular el índice Fisher fue la siguiente:

$$
\mathrm{IPF}=\sqrt{\frac{\sum \mathrm{P}_{\mathrm{n}} \mathrm{Q}_{0}}{\sum \mathrm{P}_{0} \mathrm{Q}_{0}} * \frac{\sum \mathrm{P}_{\mathrm{n}} \mathrm{Q}_{\mathrm{n}}}{\sum \mathrm{P}_{0} \mathrm{Q}_{\mathrm{n}}}}
$$

donde los valores de los precios y cantidades bases son $\mathrm{P}_{0^{\prime}}, \mathrm{Q}_{0}$ y los de los precios y cantidades mensuales, $P_{n^{\prime}} Q_{n}(16)$. Para $\mathrm{P}_{0} \mathrm{y} \mathrm{Q}_{0}$ se utilizaron el precio y la cantidad promedio comprada de cada bebida gaseosa, respectivamente.

\section{RESULTADOS}

Primero se presenta el análisis del efecto del etiquetado en los hábitos de consumo de los ecuatorianos utilizando la prueba t de Student y a continuación los resultados de los análisis de regresión, seguidos por los resultados de la prueba $t$ de Student de las diferencias en el contenido de azúcar en las bebidas gaseosas antes y después de la introducción del etiquetado.

\section{Consumo de bebidas gaseosas}

En promedio, los ecuatorianos consumieron 1,678 L/mes/per capita durante el 
período de estudio. Es importante resaltar que este valor únicamente refleja el consumo de bebidas gaseosas en casa y que el consumo de bebidas gaseosas en restaurantes y establecimientos similares no está contemplado en este análisis. Existe una clara preferencia por el consumo de CocaCola regular, que representó más de 50\% del consumo de bebidas gaseosas tanto antes como después de la introducción del etiquetado de semáforo (cuadro 2).

De 22 marcas de gaseosas evaluadas, en 10 se observó una disminución en el consumo, en 11 un aumento y en una se mantuvo el mismo nivel. De las 19 gaseosas altas en azúcar, en 7 (Coca Cola, Sprite, Fioravanti Fresa, Tropical, MAS, Oro, Fruit) hubo un descenso en el consumo promedio y en los 12 restantes, un aumento. En el grupo de bebidas gaseosas bajas en azúcar, en 2 (Coca Cola Zero y Sprite Zero) disminuyó el consumo promedio, mientras que en la otra (Coca Cola Light) aumentó.

En promedio, el consumo mensual per capita de bebidas gaseosas se redujo después del etiquetado en 0,003 L. Esta diferencia no es estadísticamente diferente de cero $(|t|=0,61 ; p=0,549)$, lo que sugiere que no hay cambios estadísticamente significativos en el consumo de bebidas gaseosas después de la introducción del etiquetado de semáforo. Al comparar el consumo entre grupos (altas y bajas en azúcar) también se aprecia una disminución en el consumo de las altas en azúcar de 0,003 L/per capita/mes $(|\mathrm{t}|=0,59 ; p=$ $0,556)$, mientras que el consumo de las bajas en azúcar aumentó 0,0003 L/per capi$\mathrm{ta} / \mathrm{mes}(|\mathrm{t}|=1,00 ; p=0,423)$. Sin embargo, ninguna de las diferencias fue estadísticamente significativa ni los valores estimados son de importancia económica.

En cuanto a los resultados de los análisis de regresión (cuadro 3), en todos los modelos se encontró un efecto significativo del precio propio (nivel de significación estadística predeterminado $\alpha=0,05$ ) y de las variables correspondientes al nivel socioeconómico. Las variables de tiempo no son significativas en los modelos de todas las bebidas gaseosas ni en

CUADRO 2. Consumo per capita de bebidas gaseosas antes y después de la introducción del etiquetado de semáforo

\begin{tabular}{llrcr}
\hline \multirow{2}{*}{ Grupo } & \multirow{3}{*}{ Litros/mes per capita } \\
\cline { 3 - 5 } Altas en azúcar & 1. Coca Cola & 0,965 & 0,880 & $-0,085$ \\
& 2. Big Cola & 0,001 & 0,003 & 0,003 \\
3. Pepsi & 0,004 & 0,005 & 0,001 \\
& 4. Fanta & 0,006 & 0,017 & 0,011 \\
5. Sprite & 0,055 & 0,051 & $-0,004$ \\
6. Fioravanti Fresa & 0,101 & 0,089 & $-0,012$ \\
7. Tropical & 0,141 & 0,117 & $-0,024$ \\
8. Inca Kola Regular & 0,002 & 0,004 & 0,002 \\
9. Coca Cola Life & 0,020 & 0,044 & 0,024 \\
10. 7UP & 0,016 & 0,021 & 0,005 \\
11. MAS & 0,114 & 0,112 & $-0,002$ \\
12. Kola Gallito & 0,002 & 0,019 & 0,017 \\
13. Oro & 0,063 & 0,061 & $-0,002$ \\
14. Manzana & 0,042 & 0,048 & 0,006 \\
15. Quintuples & 0,010 & 0,014 & 0,004 \\
16. Orangine & 0,005 & 0,006 & 0,001 \\
17. Fox Cola & 0,002 & 0,003 & 0,001 \\
18. Fruit & 0,004 & 0,002 & $-0,002$ \\
19. Barrilitos 0-Key & 0,001 & 0,001 & 0,001 \\
20. Coca Cola Zero & 0,069 & 0,068 & $-0,001$ \\
21. Coca Cola Light & 0,019 & 0,026 & 0,007 \\
22. Sprite Zero & 0,021 & 0,015 & $-0,006$ \\
23. Otras marcas & 0,002 & 0,001 & $-0,001$ \\
Cambio promedio en el consumo* & & $-0,003$ \\
Error estándar de la media & & & 0,004 \\
\hline
\end{tabular}

Fuente: elaboración propia con registros de Kantar Wolrd Panel (2016).

${ }^{*}$ Debido al redondeo de los datos, se obtiene un valor de $-0,003$, que difiere en $-0,001$ cuando se calcula con los datos del cuadro. los de las bebidas altas en azúcar, lo que indica que no hay una tendencia clara en el tiempo a mayor o menor consumo de todas las bebidas gaseosas o de las altas en azúcar específicamente. La variable de tiempo sólo fue significativa en el modelo de bebidas bajas en azúcar, lo que traduce una tendencia al alza en el consumo de estas gaseosas. En ninguno de los modelos el coeficiente de regresión de la variable del etiquetado de semáforo fue estadísticamente significativo.

Los coeficientes de regresión de los índices de precio Fisher corresponden a las elasticidades de la demanda. Por ejemplo, en el modelo de todas las bebidas gaseosas, este coeficiente sugiere que un incremento de $1 \%$ en el precio de las bebidas gaseosas disminuiría la demanda de todas las bebidas gaseosas en $0,23 \%$; por lo tanto, la demanda de bebidas gaseosas en Ecuador es inelástica con respecto al precio. Una elasticidad de la demanda respecto al precio propio muy similar se estima en el modelo para el grupo de bebidas altas en azúcar. La elasticidad de la demanda para bebidas bajas en azúcar con respecto al precio propio, aunque inelástica $(0,99)$, es mucho mayor en valor absoluto que las elasticidades de precio propio para todas las bebidas y las bebidas altas en azúcar. En suma, la demanda de bebidas bajas en azúcar es mucho más sensible a cambios en el precio propio que la demanda de bebidas altas en azúcar.

Los coeficientes de las variables dicotómicas de estacionalidad indican la presencia de un fuerte componente de estacionalidad en la demanda de bebidas gaseosas en Ecuador. Específicamente, en el primer y segundo trimestre del año la demanda promedio de todas las bebidas gaseosas es $6,6 \%\left[\left(\mathrm{e}^{0,064}-1\right) 100 \%\right]$ y $6,3 \%$ mayor $\left[\left(\mathrm{e}^{0,061}-1\right) 100 \%\right]$, respectivamente, en relación con la demanda de bebidas gaseosas en el cuarto trimestre del año. Asimismo, la demanda promedio de bebidas altas en azúcar en el primer y segundo trimestre del año es alrededor de $7 \%$ mayor respecto a la demanda en el cuarto trimestre del año. No se encontraron diferencias en el consumo entre el tercer y cuarto trimestre del año en todas las bebidas gaseosas ni en las bebidas altas en azúcar. En cuanto a las bebidas bajas en azúcar, no se encontró un efecto de estacionalidad en la demanda.

Los coeficientes de regresión de las variables dicotómicas de los grupos socioeconómicos indican que la demanda total 
CUADRO 3. Resultados de los análisis de regresión según el contenido de azúcar de las bebidas gaseosas

\begin{tabular}{|c|c|c|c|c|c|c|c|c|c|c|c|c|}
\hline \multirow{2}{*}{ Variable } & \multicolumn{4}{|c|}{ Todas las bebidas gaseosas } & \multicolumn{4}{|c|}{ Altas en azúcar } & \multicolumn{4}{|c|}{ Bajas o sin azúcar } \\
\hline & & Error estándar & $\mathrm{t}$ & $p$ & & Error estándar & $\mathrm{t}$ & $p$ & & Error estándar & $\mathrm{t}$ & $p$ \\
\hline Intercepto & 0,721 & 0,053 & 13,69 & $<0,001$ & 1,05 & 0,285 & 3,69 & $<0,001$ & $-1,821$ & 2,075 & $-0,88$ & 0,382 \\
\hline $\log (I P F G)$ & $-0,226$ & 0,061 & $-3,68$ & $<0,001$ & & & & & & & & \\
\hline $\log ($ IPFAA) & & & & & $-0,227$ & 0,064 & $-3,54$ & $<0,001$ & 0,372 & 0,488 & 0,76 & 0,448 \\
\hline $\log ($ IPFBA) & & & & & $-0,085$ & 0,069 & $-1,23$ & 0,223 & $-0,991$ & 0,485 & $-2,04$ & 0,044 \\
\hline TL & 0,011 & 0,032 & 0,36 & 0,721 & 0,01 & 0,033 & 0,31 & 0,757 & 0,171 & 0,185 & 0,93 & 0,357 \\
\hline Medio & 0,08 & 0,016 & 4,9 & $<0,001$ & 0,068 & 0,017 & 4,08 & $<0,001$ & 1,034 & 0,088 & 11,79 & $<0,001$ \\
\hline $\mathrm{T} 1$ & 0,064 & 0,027 & 2,37 & 0,02 & 0,065 & 0,027 & 2,4 & 0,018 & $-0,025$ & 0,1 & $-0,25$ & 0,806 \\
\hline T2 & 0,061 & 0,018 & 3,55 & $<0,001$ & 0,065 & 0,018 & 3,56 & $<0,001$ & $-0,035$ & 0,101 & $-0,34$ & 0,733 \\
\hline T3 & $-0,004$ & 0,017 & $-0,2$ & 0,838 & $-0,009$ & 0,018 & $-0,54$ & 0,59 & 0,039 & 0,099 & 0,4 & 0,691 \\
\hline $\mathrm{R}^{2}$ & 0,66 & & & & 0,71 & & & & 0,87 & & & \\
\hline
\end{tabular}

de bebidas gaseosas del grupo socioeconómico alto es en promedio $11 \%$ menor que la demanda del grupo socioeconómico bajo, mientras que el parámetro del grupo socioeconómico medio sugiere que este grupo consume en promedio $8 \%$ más de bebidas gaseosas que el grupo socioeconómico bajo. Resultados similares se encontraron en el modelo de la demanda para bebidas altas en azúcar. Por otro lado, el modelo para bebidas bajas en azúcar indica un fuerte incremento en el consumo promedio de bebidas al pasar del nivel socioeconómico bajo al medio y luego al alto. Con relación al grupo socioeconómico bajo, la demanda de bebidas bajas en azúcar de los grupos socioeconómicos medio y alto son 181\% y $678 \%$ mayores, respectivamente.

El coeficiente de regresión de la variable tiempo solo fue significativo en el modelo de demanda de bebidas bajas en azúcar y refleja una fuerte tendencia al incremento en el consumo promedio (alrededor de $3 \%$ de incremento mensual en el consumo).

Por último, los coeficientes de regresión de la variable del etiquetado de semáforo indican un incremento del 1,1\% en el consumo de todas bebidas gaseosas después de la introducción del etiquetado, un incremento del 1\% en el consumo de bebidas altas en azúcar y un incremento del $18 \%$ en bebidas bajas en azúcar. Sin embargo, como se mencionó anteriormente, estos efectos no son estadísticamente significativos. Por lo tanto, no se encontró evidencia estadística de un cambio en el consumo de bebidas gaseosas debido a la introducción del

CUADRO 4. Contenido nutricional antes y después de la implantación del etiquetado de semáforo

\begin{tabular}{|c|c|c|c|c|}
\hline \multirow{2}{*}{ Grupo } & \multirow{2}{*}{ Descripción } & \multicolumn{3}{|c|}{$\mathrm{g} / 100 \mathrm{ml}$ de producto } \\
\hline & & Antes & Después & Cambio en el contenido de azúcar \\
\hline \multirow[t]{11}{*}{ Altas en azúcar } & 1. Coca Cola & 10,48 & 10,68 & 0,20 \\
\hline & 2. Big Cola & 9,40 & 10,23 & 0,83 \\
\hline & 3. Pepsi & 11,35 & 10,70 & $-0,65$ \\
\hline & 4. Fanta & 12,45 & 13,81 & 1,36 \\
\hline & 5. Sprite & 10,06 & 8,85 & $-1,21$ \\
\hline & 6. Fioravanti Fresa & 10,53 & 7,50 & $-3,03$ \\
\hline & 7. Tropical & 10,00 & 9,14 & $-0,86$ \\
\hline & 8. Inca Kola Regular & 7,74 & 0 & $-7,74$ \\
\hline & 9. Cola Gallito & 7,43 & 7,60 & 0,17 \\
\hline & 10. Fox Cola & 12,20 & 10,32 & $-1,88$ \\
\hline & 11. Barrilitos 0-Key & 0 & 0 & 0 \\
\hline \multirow[t]{5}{*}{ Bajas en azúcar } & 12. Coca Cola Zero & 0 & 0 & 0 \\
\hline & 13. Coca Cola Light & 0,20 & 0 & $-0,20$ \\
\hline & 14. Sprite Zero & 0 & 0 & 0 \\
\hline & \multirow{2}{*}{\multicolumn{2}{|c|}{$\begin{array}{l}\text { Cambio promedio del contenido de azúcar } \\
\text { Error estándar de la media }\end{array}$}} & & $-0,93$ \\
\hline & & & & 0,60 \\
\hline
\end{tabular}

Fuente: Elaboración propia con registros de la Agencia Nacional de Regulación, Control y Vigilancia Sanitaria (ARCSA), Ecuador, 2016.

etiquetado de semáforo durante el primer año de su implementación.

\section{Diferencias en el contenido de azúcar de las bebidas gaseosas}

Del total de 14 marcas de bebidas gaseosas evaluadas en esta etapa, siete (Pepsi, Sprite, Fioravanti Fresa, Tropical, Inca Cola, Fox Cola y Coca Cola Light) tuvieron un contenido de azúcar menor después de la implementación del etiquetado de semáforo, cuatro (Coca Cola, Big Cola, Fanta y Cola Gallito) tuvieron un contenido de azúcar mayor después de la introducción del etiquetado, y solamente tres (Barrilitos O-Key, Coca Cola Zero y Sprite Zero) no cambiaron su formulación en cuanto al contenido de azúcar (cuadro 4). Sin embargo, tan solo dos de ellas (Fioravanti e Inca Kola) mostraron cambios que resultaron en un cambio de color del etiquetado de semáforo de amarillo o rojo a verde.

En promedio, el contenido de azúcar de todas las bebidas gaseosas se redujo 0,93 gr/100 $\mathrm{ml}$ (aproximadamente el 13\% del contenido de azúcar antes del etiquetado), aunque esta diferencia no es estadísticamente significativa cuando se 
aplica la prueba $t$ de Student bilateral $(|\mathrm{t}|=1,54 ; p=0,146)$ y sí lo es $(\mathrm{al} 10 \%)$ si se usa una prueba unilateral $(\mathrm{t}=-1,54$; $p=0,073)$. Asimismo, el grupo de bebidas de contenido alto en azúcar tuvo una disminución después del etiquetado $(1,16 \mathrm{gr} / 100 \mathrm{ml})$ que no fue estadísticamente significativa si se usa una prueba bilateral $(|\mathrm{t}|=1,54 ; p=0,1547)$, si bien lo es al $10 \%$ si usa una prueba unilateral. No se aplicó la prueba de hipótesis sólo para bebidas bajas en azúcar, dado que la muestra solo contiene tres bebidas, pero en promedio este grupo de bebidas experimentaron una reducción $(0,07$ gr $/ 100$ $\mathrm{ml}$ ) en su contenido de azúcar. (El uso de un $10 \%$ de nivel de significación estadística se justifica porque el tamaño de la muestra es pequeño. Las pruebas de hipótesis unilaterales se fundamentan en que las expectativas a priori en este estudio eran que la introducción del semáforo disminuiría el consumo y el contenido de azúcar en las bebidas gaseosas.)

\section{DISCUSIÓN}

Los resultados de este estudio son similares a los encontrados en estudios anteriores en los cuales se observó que los hábitos de compra de los consumidores no cambian significativamente por la presencia del etiquetado de semáforo $(9,10)$. Además, los resultados de los estudios cualitativos realizados en Ecuador ayudan a explicar los resultados del presente estudio, porque los tres estudios revelaron que los consumidores consideran que sus hábitos de consumo no se han visto afectados por el etiquetado (11-13).

No hay estudios similares que hayan estimado la elasticidad precio de la demanda de bebidas gaseosas en Ecuador. Sin embargo, en un estudio en el cual se estimaron elasticidades de precio y efecto del nivel socioeconómico en el consumo

Watson W, Kelly B, Debra H, Hughes C, King L, Crawford J, et al. Can front-of-pack labelling schemes guide healthier food choices? Australian shoppers' responses to seven labelling formats. Science Direct. 2014;92(1):2-3.

2. World Health Organization. Fiscal policies for diet and prevention of noncommunicable diseases: technical meeting report. WHO Library Cataloguing-in-Publication Data. 2016;4(1):14-8.

3. Banco Mundial. Países de ingreso mediano-alto. Washington, DC: Banco Mundial; de bebidas azucaradas en general se observó que la demanda de estos productos es elástica con respecto al precio (17). El presente estudio contrasta con el anterior porque la elasticidad de la demanda de bebidas gaseosas con respeto al propio precio es inelástica; sin embargo, cabe recalcar que los estudios no son completamente comparables ya que en ese estudio se incluyen todos los tipos de bebidas azucaradas y el presente únicamente bebidas gaseosas (17).

Como conclusiones cabe decir que no se encontraron pruebas empíricas de que la implementación del etiquetado de semáforo haya cambiado los hábitos de consumo de bebidas gaseosas en Ecuador durante el primer año de implementación de la políti$\mathrm{ca}$, ni en general ni en las gaseosas altas en azúcar específicamente. Sin embargo, se encontró evidencia de que la implementación del etiquetado de semáforo si resultó en una disminución importante del contenido de azúcar de las bebidas gaseosas en Ecuador (13\% menos).

Adicionalmente se encontró que, durante el período observado, el consumo de gaseosas altas en azúcar es mayor en los grupos socioeconómicos bajos y medios, que la época de mayor consumo de gaseosas se produce en el primer y segundo trimestre del año y que la demanda de las bebidas gaseosas bajas en azúcar es mucho más elástica con respecto al propio precio que la demanda de las bebidas altas en azúcar, aunque ambas son inelásticas.

Debido al mayor consumo de bebidas gaseosas por los grupos socioeconómicos medio y bajo y el mayor consumo durante los primeros dos trimestres del año, se recomienda que las campañas de publicidad de información sobre el uso correcto del etiquetado de semáforo se deben dirigir principalmente a estos grupos socioeconómicos durante estas épocas del año.

\section{REFERENCIAS}

2018. Disponible en: http://datos.bancomundial.org/nivel-de-ingresos/ingresomediano-alto Acceso el 1 de Julio de 2017.

4. Freire WB, Ramirez-Luzuriaga MJ, Belmont P, Mendieta MJ, Silva-Jaramillo MK, Romero N, et al. Encuesta Nacional de Salud y Nutrición de la Población Ecuatoriana de cero a 59 años. ENSANUTECU 2012. Vl 1. Quito: Ministerio de Salud Pública, Instituto Nacional de Estadisticas y Censos; 2014.

5. Ministerio de Industria y Productividad. Reglamento Técnico Ecuatoriano RTE
Este estudio tiene tres limitaciones importantes. Primero, no se evaluó el efecto del etiquetado de semáforo después de diciembre de 2015, porque en 2016 se aprobó un impuesto a las bebidas gaseosas. Se decidió limitar el período del estudio para observar el efecto del etiquetado de semáforo por sí solo y no en conjunto con el del impuesto. Esto no implica que el etiquetado no haya modificado el consumo a partir del segundo año de implementación, sino simplemente que no se puede cuantificar. Segundo, hay otras variables que pueden afectar el consumo de bebidas gaseosas además de las analizadas en este estudio, como los hábitos saludables. Sin embargo, por la naturaleza agregada de los datos utilizados en este estudio, es imposible incluir variables personales que afecten el consumo de las bebidas gaseosas. Tercero, los resultados contemplan únicamente el consumo de bebidas gaseosas en el hogar. El consumo de bebidas gaseosas fuera del hogar debe abordarse en extensiones de esta investigación. Por último, los resultados se circunscriben a las bebidas gaseosas y no se pueden extrapolar a otras categorías de producto.

Financiación. Los fondos para adquirir la base de datos de Kantar World Panel fueron proporcionados por el Centro Internacional para la Excelencia en la Industria Alimentaria (ICFIE).

Conflictos de interés. Los autores declaran no tener conflictos de interés

Declaración. Las opiniones expresadas en este manuscrito son responsabilidad de los autores y no reflejan necesariamente los criterios ni la política de la RPSP/ PAJPH y/o de la OPS.
INEN 022 "Rotulado de productos Alimenticios procesados, envasados y empaquetados" (Etiquetado de productos alimenticios procesados). Quito: MIP; 2013.

6. U.S. Department of Health and Human Services,U.S. Food and Drug Administration. Etiquetado de información nutricional: Guía para la industria: Etiquetado de nutrición. Washington, DC, Bethesda, MD: U.S. Department of Health and Human Services, FDA; 2018. Disponible en: https://www. fda.gov/Food/GuidanceRegulation/ 
GuidanceDocumentsRegulatory Information / Labeling Nutrition / ucm247927.htm Acceso el 27 de enero de 2018.

7. Malam S, Clegg S, Kirwan S, McGinigal S, BMRB Social Research. Comprehension and use of UK nutrition signpost labelling schemes. London: Food Standards Agency; 2009. Disponible en: http://www.ampelcheck.de/files/000001/1397_abschlussbericht_der_unabhaengigen_exper.pdf. 2009 Acceso el 5 de julio de 2017.

8. Kelly B, Hughes C, Chapman K, Chun-Yu Louie J, Dixon H, Crawford J, et al. Consumer testing of the acceptability and effectiveness of front-of-pack food labelling systems for the Australian grocery market. Health Promotional Intl. 2009;24(2):120-9.

9. Sacks G, Rayner M, Swinburn B. Impact of front-of-pack 'traffic-light' nutrition labelling on consumer food purchases in the UK. Health Promotion Intl. 2009;24(4):344-52.

10. Sacks G, Tikellis K, Millar L, Swinburn B. Impact of 'traffic-light' nutrition information on online food purchases in Australia.
Australian New Zealand J Public Health. 2011;35(2):122-6

11. De Souza J. Análisis del impacto de las etiquetas de alimentos procesados. [Tesis doctoral.] Quito: Universidad San Francisco de Quito; 2015:52-3.

12. Maya M. Etiquetado de semáforo; Estudio del hábito de compras en jugos procesados en el barrio de San Carlos de la ciudad de Quito. [Tesis demaestría.] Quito: Universidad Andina Simón Bolívar; 2015:77-9.

13. Freire WB, Waters WF, Rivas-Mariño G, Nguyen T, Rivas P. A qualitative study of consumer perceptions and use of traffic light food labelling in Ecuador. Public Health Nutrition. 2016;20(5):805-13.

14. Díaz AA, Veliz PM, Rivas-Mariño G, Vance Mafla C, Martínez Altamirano LM, Vaca Jones C. Etiquetado de alimentos en Ecuador: implementación, resultados y acciones pendientes. Rev Panam Salud Publica. 2017;41:e54.

15. Deaton A, Muellbauer J. Economics and consumer behavior. Cambridge: Cambridge University Press; 1980.
16. Organization for Economic Cooperation and Development. Glosary of statistical terms: Fisher's ideal index (Price). Disponible en: http://stats.oecd.org/ glossary/detail.asp?ID=988 Acceso el 20 de abril de 2017.

17. Paraje G. The Effect of Price and SocioEconomic Level on the Consumption of Sugar-Sweetened Beverages (SSB): The Case of Ecuador. PLOSone. 2016. doi. org / 10.1371/journal.pone.0152260 Disponible en: http://journals.plos.org/ plosone / article?id=10.1371/journal. pone.0152260 Acceso el 20 de septiembre de 2018.

Manuscrito recibido el 2 de febrero de 2018 Aceptado para publicación, tras revisión, el 28 de agosto de 2018 .

Objective. To evaluate the effect of "traffic-light" nutritional labeling on the purchase of soft drinks and on their nutritional content in Ecuador.

Methods. Two databases were used: the first was provided by Ecuador's National Agency for Health Regulation, Control, and Surveillance; the second was obtained

Effect of traffic-light labeling on nutritional content and on consumption of carbonated beverages in Ecuador

Keywords from the international market research company Kantar World Panel. A before/after study was conducted of introducing traffic-light labeling, using Student's t-tests to evaluate changes in average nutritional content and the purchase of soft drinks. Multiple linear regression methods were used to evaluate changes in purchases resulting from the introduction of traffic-light labeling.

Results. After labeling, average per capita consumption of soft drinks declined by $0.003 \mathrm{~L} /$ month - a small change with respect to average per capita consumption of $1.678 \mathrm{~L} /$ month. The results of the Student's t-tests indicated that this difference was not statistically significant. Furthermore, the regression analyses found no empirical evidence that traffic-light labeling affected soft drink consumption. With regard to sugar content, an average reduction of $0.93 \mathrm{gm} / 100 \mathrm{ml}$ of beverage was observed.

Conclusions. No empirical evidence was found that implementation of traffic-light labeling changed soft-drink purchase habits in Ecuador, but there is evidence that it reduced sugar content in carbonated beverages in the country.

Carbonated beverages; obesity; health policy; Ecuador. 
RESUMO Objetivo. Avaliar o efeito da rotulagem nutricional com modelo de semáforo na compra e no teor nutricional dos refrigerantes no Equador.

Métodos. As informações foram obtidas do banco de dados da Agência Nacional de Regulamentação, Controle e Vigilância Sanitária do Equador e do banco de dados da

Efeito da rotulagem nutricional com modelo de semáforo no consumo de refrigerantes no Equador

Kantar World Panel, empresa internacional de pesquisa de mercado. Foi feita uma análise do tipo antes-depois da introdução da rotulagem com modelo de semáforo. Foram usados testes $t$ de Student para avaliar a variação nas médias do teor nutricional e na compra de refrigerantes e métodos de regressão linear multivariada para avaliar a variação na compra com a introdução da rotulagem com modelo de semáforo. Resultados. Foi observada uma redução de 0,003 litro/mês no consumo médio per capita de refrigerantes com a introdução da rotulagem, que representa um valor pequeno em relação ao consumo médio per capita (1,678 litro/mês). Os resultados dos testes $t$ de Student indicaram que esta diferença não foi estatisticamente diferente de zero. As análises de regressão também não forneceram evidências empíricas de que a rotulagem com modelo de semáforo tenha influído no consumo de refrigerantes. Com relação ao teor de açúcar, foi verificada uma redução média de 0,93 g por $100 \mathrm{ml}$ de bebida.

Conclusões. Não foram observadas evidências empíricas de que a implantação da rotulagem nutricional com modelo de semáforo alterou os hábitos de compra de refrigerantes, porém houve uma diminuição no teor de açúcar destas bebidas no Equador.

Palavras-chave Bebidas gaseificadas; obesidade; política de saúde; Equador. 